\title{
IMAGING PERFORMANCE AND TESTS
}

\section{OF SOFT X-RAY TELESCOPES}

E. Spiller, R. McCorkje, J. Wilczynski

IBM T.J. Watson Research Center, P.O. Box 218, Yorktown Heights, NY 10598

L. Golub, G. Nystrom

Center for Astrophysics, Cambridge, MA 02138

\section{Peter Z. Takacs}

Brookhaven National Laboratory, Upton, NY 11973

C. Welch

Lockheed Missile and Space Company, P.O. Box 970, Las Cruces, NM 88004

*This research was supported by the U. S. Department of Energy: Contract No. DE-AC02-76CH00016. 
DISCLAIMER

This report was prepared as ari acoount of work sponsored by an agency of the United States Government. Neither the United States Government nor any agency thereof, nor any of their employes, makes any warranty, express or imiplied, or assumes any legal liability or responsibility for the accuracy, completeness, or usefulness of any information, apparatus, product, or process disclosed, or represents that its use would not infringe privately owned rights. Reference herein to any specific commercial product, process, or service by tiade name, tradeinark, manufacturer, or otherwise does not necessarily constitute or imply its endorsement, recommendation, or favoring by the United States Government or any agency thereof. The views and opinions of authors expressed herein do not necessarily state or reflect those of the United States Government or any agency thereof. 


\title{
Imaging Performance and Tests of Soft X-Ray Telescopes
}

\author{
E. Spiller, R. McCorkle, J. Wilczynski, \\ IBM T. J. Watson Research Center, P.O. Box 218, Yorktown Heights, NY 10598
}

Center for Astrophysics, Cambridge MA 02138

P. Takacz, Brookhaven National Laboratory, Upton NY 11973

C. Welch,

Lockheed Missile and Space Company, P.O. Box 970, Las Cruces N.M 88004

\begin{abstract}
Photos obtained during $5 \mathrm{~min}$. of observation time from the flight of our 10 inch normal incidence soft $x$-ray $(\lambda=63.5 \AA)$ telescope on September 11,1989 are analyzed and the data are compared to the results expected from tests of the mirror surfaces. These tests cover a range of spatial periods from 25 $\mathrm{cm}$ to $1 \mathrm{~A}$. The photos demonstrate a reduction in the scattering of the multilayer mirror compared to a single surface for scattering angles above 1 arcmin, corresponding to surface irregularities with spatial periods below $10 \mu \mathrm{m}$. Our results are used to predict the possible performance of future flight.s. Sounding rocket observations might be able to reach a resolution around 0.1 arcsec. Higher resolutions will require flights of longer durations and improvements in mirror testing for the largest spatial periods.
\end{abstract}

\section{INTRODUCTION}

Diffraction defines the resolution limit of optical instruments for visible light and the capabilities of fabricating diffraction limited visible light optics has existed for about a century. Multilayer coated mirrors for soft $x$-rays have permitted the fabrication of optical elements (telescopes and microscopes) for wavelengths that are 100 times shorter with a corresponding improvement in the theoretically possible resolution. Even a modest size telescope with $10 \mathrm{~cm}$ aperture has the potential for 0.01 arcsec resolution. The fabrication, assembly, testing and use of such an instrument, however, poses some chailenges that are beyond our present technology.

Multilayer mirror telescopes have been flown on sounding rockets $[1,2]$ and have produced $x$-ray images of the sun with a resolution better than 1 arcsec. We discuss in this paper the present limits on the performance of such telescopes, using our 10 inch telescope for $\dot{\lambda}=63 \AA$ and its Sept. 11, 1989. 
flight as a specific example. Areas where progress is needed are defined, and estimates are given on the improvements that we expect in future missions.

\section{MIRROR OPTICS}

The height deviations $z(x, y)$ of the actual surface of a mirror from its perfect shape have to be drastically smaller for soft $x$-rays than for visible light. The same is true for all the boundaries within the multilayer coating. The Fourier transform of the deviation $z(\vec{r}), \vec{r}=(x, y)$ defines the twodimensional power spectrum of the surface errors [3-6]

$$
\underset{\lim _{2}(\vec{f})}{S_{A} \rightarrow \infty}=\frac{1}{A}\left|\int_{A} z(\vec{r}) e^{2 \pi i \vec{f} \cdot \vec{r}} \overrightarrow{d r}\right|^{2}
$$

From one-dimensional scans $z(x)$ of the surface one obtains the one-dimensional power spectral density

$$
\underset{\lim L \rightarrow \infty}{S_{I}\left(f_{x}\right)}=\frac{2}{L}\left|\int_{0}^{L} z(x) e^{2 \pi i f_{x} x} d x\right|^{2},
$$

where $A, L$ are the area or length of the scans, $\vec{f}=\left(f_{x}, f_{y}\right)$ is the 2-D vector of spatial frequencies, and $r_{x}=1 / \Lambda_{x}, \Lambda_{x}=$ spatial period in the surface. See Ref. [4] for the formulas that connect $S_{1}$ and $S_{z}$.

All statistical properties of a surface can be obtained from the power spectral density. The rms sur. face roughness is given by

$$
\sigma^{2}=\int_{0}^{\infty} S_{1}\left(f_{x}\right) d f_{x}
$$

or

$$
\sigma^{2}=2 \pi \int_{0}^{\infty} S_{2}(\cap) f d f
$$

Figure 1 contains a list of methods that are used to characterize optical surfaces. Each method characterizes a surface over a limited band of spatial frequencies or periods and the bands for different methods are indicated in the figure together with their stinsitivity. For a high quality, near diffraction limited $x$-ray mirror, the entire range of spatial freqtiencies from periods in the IA-range up to the size of the optics is importan?. The fine features in the surface roughness reduce the reflectivity of a mirror, and the roughness should be smaller than about $1 ; 10$ of the multilayer period for good reflectivity. The reduction of the reflectivity is described by the well known Debye-Waller factor [7-9]. The roughness value $\sigma$ obtained from an $x$-ray reflectivity measurement contains all spatial frequencies from a lower limit (determined by the detector aperture in a reflectometer) to infinity (or in practice the value defined by atomic structure).

Total Integrated Scattering (TIS) with visible light also yields a value of $\sigma$ integrated over a large frequency band with a resolution in the $1 \AA$ range. However, the band of frequencies included in this measurement, is of lesser importance for x-ray optics. The methods below the dotted line in Fig. 1 permit measurement of the power spectral density for each spatial frequency separately. Electron 
microscopy $[10,11]$ and tunnel microscopy [12] cover the high spatial frequencies and can even show each individual boundary in a sliced multilayer coating. X-ray scattering represents a direct Fourier analysis of a surface, and the intensity of the scattered light is completely determined by the structure as obtained by the microscopes. However, additional work is still required to connect the results between the two methods.
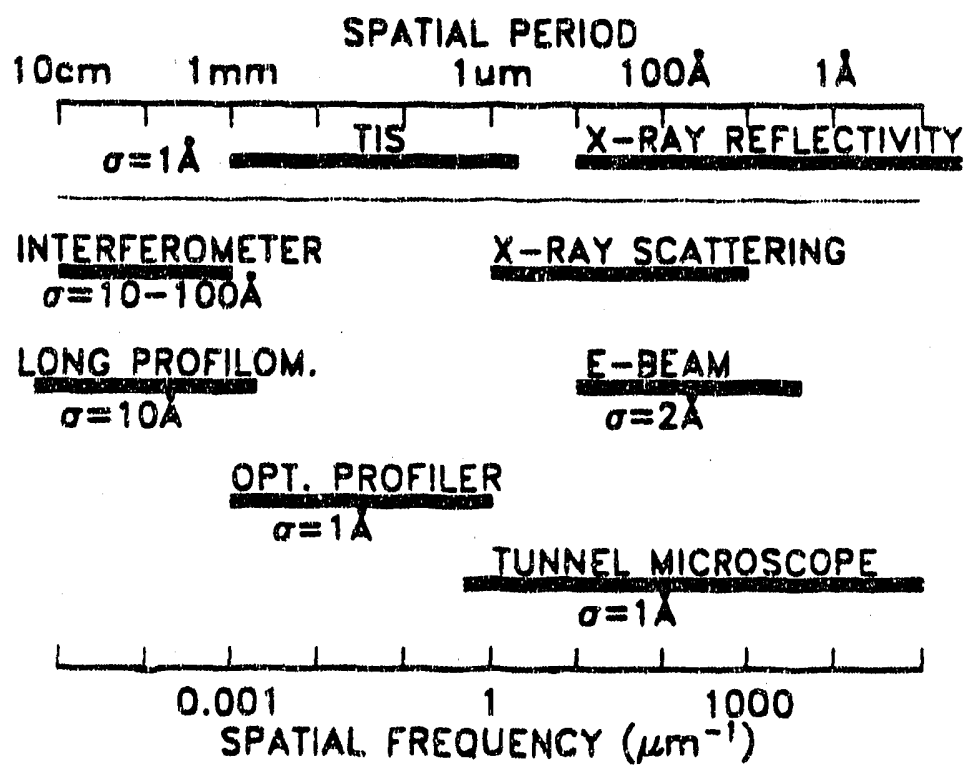

Fig. 1. Methods to characterize mirror surface for different ranges of spatial frequencies. Total integrated scattering (TIS) and $X$-ray rellectivity measurements give only the integral over all spacial frequencies within their range, while the other methods can measure the power spectrum of the surface errors at each spatial frequency within their range.

The topography of a surface at the highest spatial frequencies is mostly determined by the growth processes in the coating structure and by the size of the atoms. The metrology for these highest spatial frequencies has drastically advanced in recent years. Figure 2 shows the surface topography of Co-C multilayer coatings as obtained with a scanning tunnel microscope. At the left is the surface of the coating used in the 10 inch telescope flown in 1989, while the right picture represents the top surface of a coating, where the boundaries have been ion polished [13]. Ion polishing has reduced the roughness mainly for the spatial periods in the $100-500 \AA$ range. The roughness values obtained from reflectivity measurements and those derived from the tunnel microscope are in quantitative agreement. We also hope to obtain tesults from $x$-ray scattering and from microscopy of sliced coatings. While it still may be a problem to fabricate a surface with the required quality, the characterization is not a serious problem for the highest spatial frequencies.

There have also been advances in the characterization of surfaces at the low spatial frequencies. Optical interferometry with phase musulation can have a height resolution in the $1 \AA$-range and commercial instruments, which are easy to use, have been developed. Figure 1 shows that instruments which cover the entire range are now available and we have used the instruments to characterize our telescope optics. The results of these tests are summarized in Fig. 3, where the 1-D power spectrum $S_{1}$ is plotted over 10 decades of spatial frequencies. The curves labeled "TUNNEL" are obtained from about 30 pictures such as those in Fig. 2; WYKO represents measurements with a commercial optical profilometer [14], BNL is the LONG RANGE PROFILOMETER at the Brookhaven National Lab. oratory [15] and ZYGO is a commercial Fizeau interferometer [16] (Fig. 4). The top scale in Fig. 3 


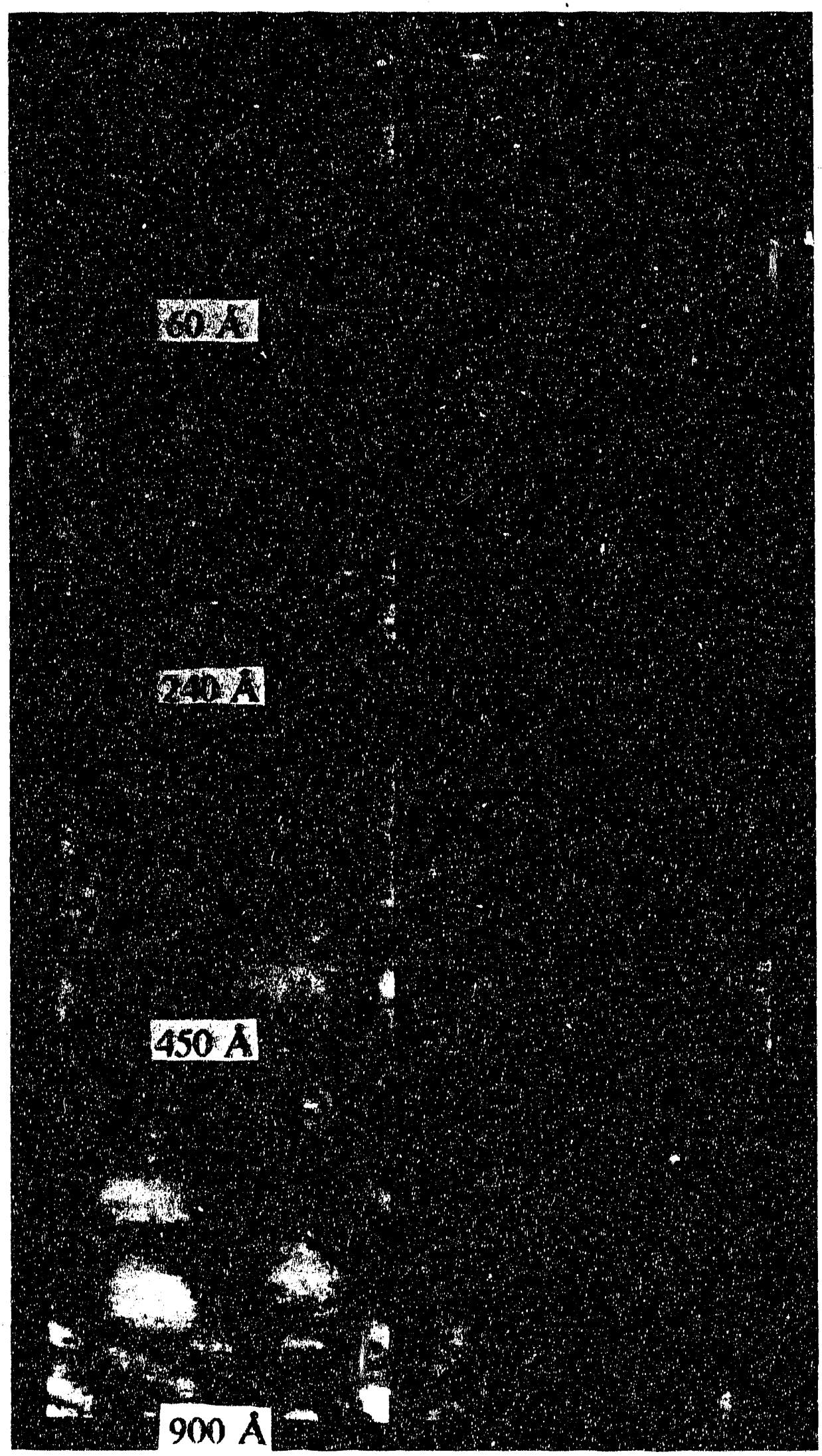

Fig. 2. Tunnel micrographs of the 140 layer Co-C coating as used for the 10 inch telescope (left) and of a similar Co-C coating, where the layers have been smoothed by ion polishing. The size of the pictures in each row is indicated. 


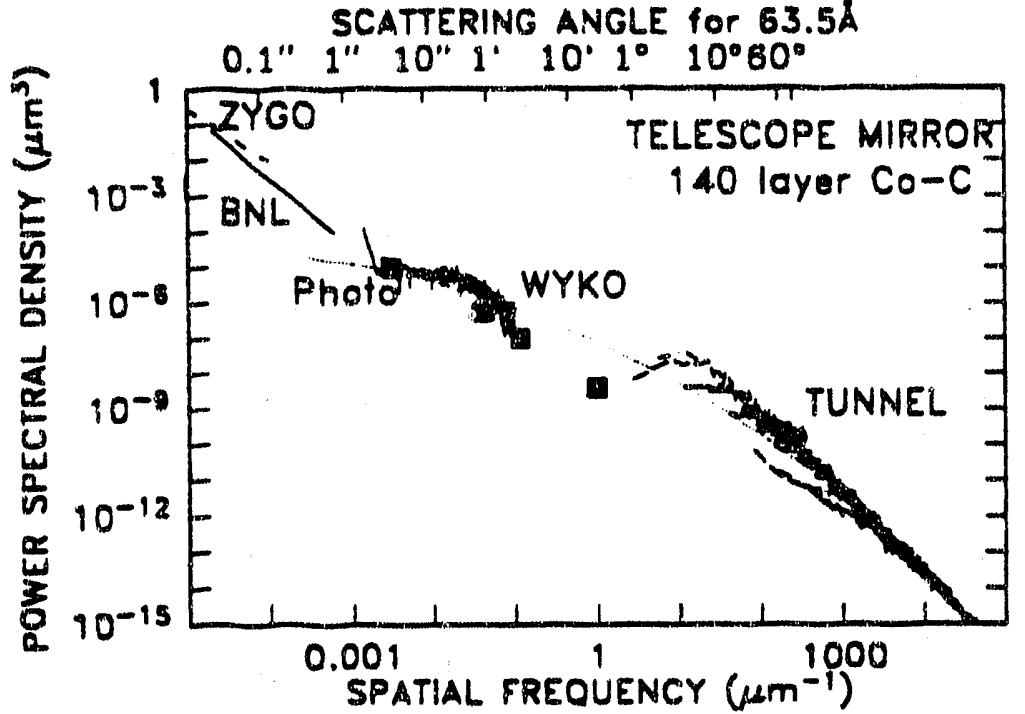

Fig. 3. Power spectral density $S_{1}(\cap)$ of the 10 inch telescope mirror obtained by various methods. Tunnel: Data derived from tunnel micrograph like those shown in Fig. 2. WYKO: data obtained from an optical profilometer. BNL: data from the long range profilometer at the Brookhaven National Laboratory. ZYGO: data from a commercial Fizeau interferometer. The dolced line is a smooth curve through the data. The bleck square arc derived from an analysis of the $x$-ray photo of the sun (see Fig. 5) and show the reduction in scattering in a multilayer structure compared to a single surface. The scattering angle on the top scale are for normal incidence.

Table 1 Contribution to roughness and figure errors $\sigma$ from different spatial frequencies ranges in the power spectral density of the telescope mirror obtained from the dotted curve in Fig. 3. The second column give the range of scattering angles $\Delta \theta$ and the fourth column is the fraction of the reflected light that would be scattered by a single surface into this angular range.

\begin{tabular}{|c|c|c|c|}
\hline frange $\left(\mu \mathrm{m}^{-1}\right)$ & $\Delta \theta$ & $\sigma(\AA)$ & $1-\exp -\left(\frac{4 \pi \sigma}{\lambda}\right)^{2}$ \\
\hline $10^{-4.4}-10^{-3}$ & $0.1^{\prime \prime}-1^{\prime \prime}$ & 1.3 & 0.06 \\
$10^{-3}-10^{-2}$ & $1^{\prime \prime}-10^{\prime \prime}$ & 2.5 & 0.21 \\
$10^{-2}-10^{-1}$ & $10^{\prime \prime}-100^{\prime \prime}$ & 3.8 & 0.43 \\
$0.1-1$ & $1.7^{\prime}-17^{\prime}$ & 4.5 & 0.55 \\
$1 .-10$ & $17^{\prime}-3^{\circ}$ & 4.1 & 0.48 \\
$10-100$ & $3^{\circ}-30^{\circ}$ & 2.9 & 0.28 \\
\hline
\end{tabular}

gives the scattering angles $\theta_{3}$ for normal incidence and $\lambda=63.5 \AA, \sin \theta_{s}=\lambda / \Lambda$. The black squares have been obtained by analyzing the pictures (Fig. 5) obtained during the fight in 1989, and the dotted line is a smooth interpolation of most of the data. The most difficult region is at the lowest spatial frequencies where measurernents over large distance along the mirror surface are required. We believe that the two curves (ZYGO and BNL) in Fig. 3 do not represent the quality of the mirror but the noise level of the instruments. For the tests with the ZYGO interferometer (Fig. 4) most of this noise is due to vibrations and nuctuations in the air path. We think that this noise can be reduced by averaging over a large number of interferograms. Testing of the optics in a vacuum tank will probably be required, if one wants to predict the performance below the 0.1 arcsec range. The performance of the BNL profilometer with a much shorter air path is presently limited by amplifier noise, which we expect to reduce in the near future. 

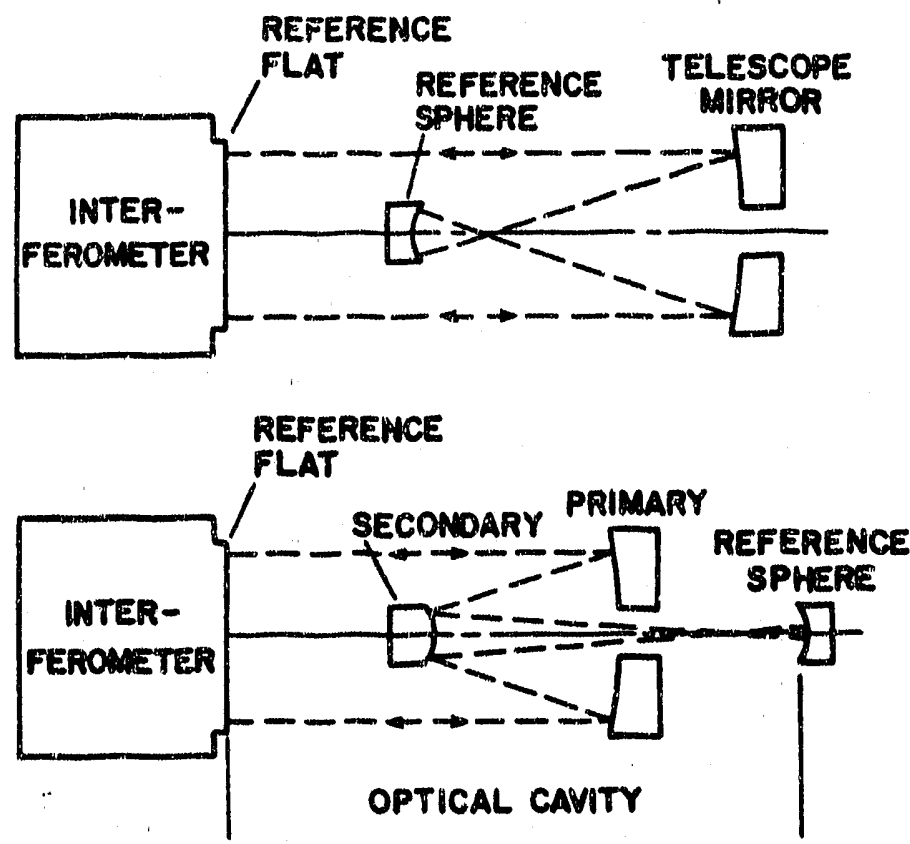

Fig. 4. Setup for the test of a prime focus and a Ritchie Chretien telescope in a Fizeau interferometer.

Fig. 5. X-ray picture of the sun obtained from the 10 inch telescope on Sept. 11, 1989. The contrast in the picture (exposure intensity in dark areas compared to close-by bright areas) has been used to obtain the points in Fig. 3.

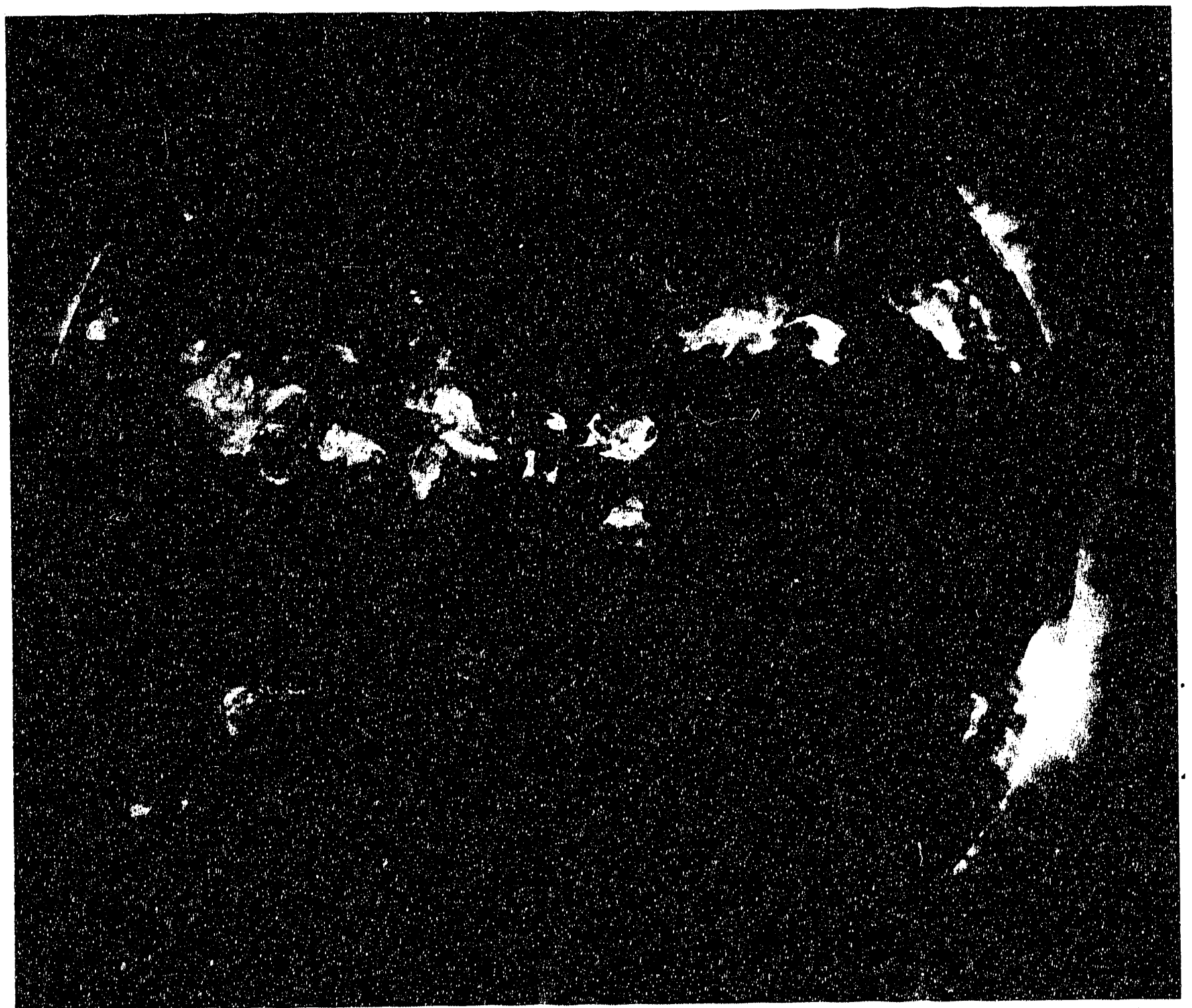



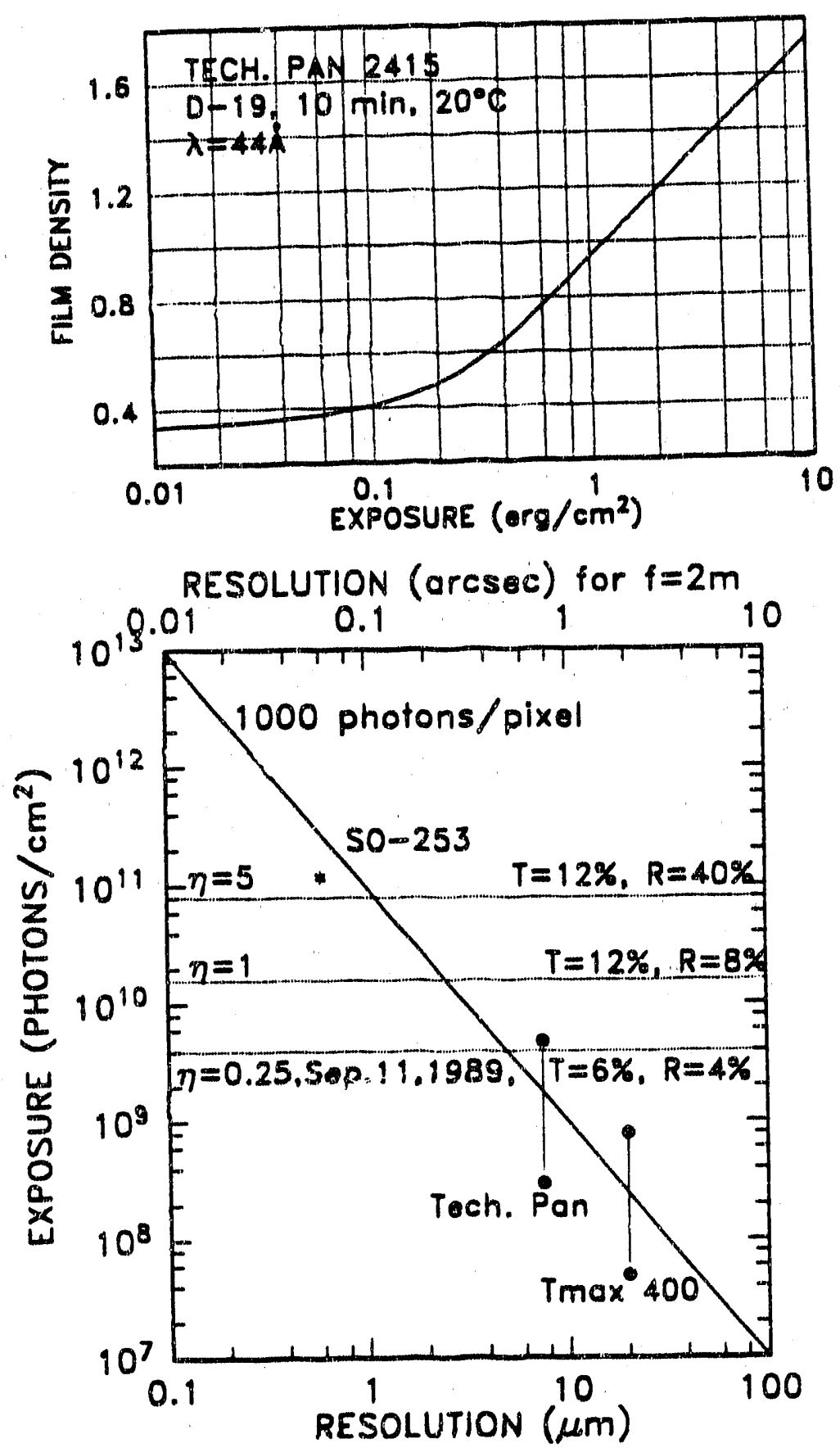

We can for a single surface mirror estimate the size and intensity of the halo of scattered light around each image point, by integrating Eq. (4) over a finite frequency band to obtain an effects s value for $\sigma$ and by estimating the fraction SC of the incident light scattered into a small ring around the image point from

$$
S C=1-\exp \left[-\left(\frac{4 \pi \sigma}{\lambda}\right)^{2}\right]
$$

The result of this estimate derived from the dotted curve in Fig. 3 is given in Table 1. The data predict a large amount of scattering for the larger scattering angles above 10 arcsec which is not con- 
sistent with the quality of the pictures obtained (Fig. 5). The discrepancy can be expliuned, if one assumes that the roughnesses of the different boundaries in the multilayer become un-correlated for high spatial frequencies above $1 / \mu \mathrm{m}$. In this case the contributions to scattering from different bolindaries are added with random phases, and the effective roughness of a multilayer is be reduced by nearly a factor $1 / \sqrt{N}(N=$ number of layers) over that of the single boundary $[5,6,9]$, resulting in a drastically reduced amount of scattering. Our Fig. 5 is the first experimental verification of this effect for soft $x$-rays.

We want to note that the points in Fig. 3, derived from the $x$-ray photo of Fig. 5, represent upper limits. Using the measured density versus exposure curve of the film (Fig. 6), we select dark areas close to very bright areas in the photo. Measuring the exposure intensity in the bright and the dark areas and assuming that all the intensity in the darker section is produced by scattering from the surroundings, we obtain a $\sigma$ value and an estimate for the power spectral density. The actual values might be lower, because some $x$-ray flux might also emanate from the darker regions in the corona.

\section{PHOTON FLUX AND DETECTOR}

About 1000 photons per pixel have to be collected to obtain a picture with about 100 gray levels. Photos with increased resolution have more pixels and therefore require a higher exposure. For sounding rockets the size of the optics and the observation time are limited and define an effective linit on the ultimate resolution. We used modified Kodak Technical Pan and $T_{\max } 400$ film for our flight and have plotted in Fig. 7 the range of exposure intensities in a $1 \mathrm{~min}$. and a $10 \mathrm{sec}$. exposure in the image of active regions of the sun at the spatial resolution of those films. We have also plotted an estimate for film of drastically higher resolution, holographic film SO253.

The exposure level required for 1000 photons/pixel is plotted as a function of spatial resolution in the film plane (bottorn) or angular resolution of a telescope with $2 \mathrm{~m}$ focal length (top). The horizontal dotted line at $4 \times 10^{9}$ photons $/ \mathrm{cm}^{2} \mathrm{~min}$ represents the exposure level obtained for the brighter parts of the active region during our 1989 flight within 1 minute. We cannot expect a drastic increase in resolution in future tlights unless we increase the number of photons collected. The collection efficiency $\eta$ of a telescope is defined as

$$
\eta=\left(\frac{A P}{I M}\right) \cdot T \cdot R
$$

where AP is the area of the entrance aperture, IM is the area of the sun's image on the film, $T$ is the $x$-ray transmission of all filters in the telescope and $R$ the mirror reflectivity; we had $\eta=0.25$ in 1989 . By using improved filters [17] and a mirror with higher reflectivity produced with ion polishing [13] we expect a value $\eta=1$ for the next flight with a resolution potential of 0.3 arcsec. A collection efficiency of 5 would be required for a resolution of 0.1 arcsec for $1 \mathrm{~min}$ observation time, and this would require multilayer mirrors with boundaries of $\sigma<2.5 \AA$, or larger mirrors.

An improvement in the resolution of a telescope requires a detector that matches the required resolution and sensitivity. There is at the present time a gap in the commercially available films in the 
range from 400-1000 lines/mm resolution. Matching the instrument to existing photographic films requires a telescope with larger focal lengths. However, $r=2 \mathrm{~m}$ is close to the limit of the payload lengths of a sounding rocket, and a folded system like a Ritchie-Chretien as we used in our first flight suffers from the reflection loss in the second mirror.

We conclude that the available photon flux will permit us to reach a resolution around 0.3 arcsec in the next flight and that 0.1 arcsec represents the optimistic limit that one could hope to achieve with a sounding rocket at $\lambda=63.5 \AA$. Some emission lines at longer wavelengths are more than a factor 10 stronger than the FeXVI line at $\lambda=63.5 \AA$ and should easily provide the photon flux required for 0.1 arcsec resolution. However, the photos [1] obtained at these wavelengths up to now have a lower resolution than those obtained at $\lambda=63.5 \AA$.

\section{MECHANICAL STABILITY AND ALIGNMENT}

For observations from sounding rockets the telescope has to be assembled, aligned and focussed on the ground in such a way that the changes in environment and the severe vibrations during launch do not change any critical parameter. For the Ritchie-Chretien telescope the position of the secondary mirror is the most critical parameter; the error in this position has to be within $\pm 4 \mu \mathrm{m}$ for a resolution of 0.1 arcsec for our design. For the prime focus telescope as used in 1989 ( $\mathrm{f}=2 \mathrm{~cm}$, diameter $25 \mathrm{~cm})$ the film has to be within $\pm 8 \mu \mathrm{m}$ of the correct focus for the same resolution.

The mechanical design of our payload has been described previously [18]. The secondary mirror, or the camera in the prime focus geometry, are held in position by a carbon fiber/epoxy tube with a thermal expansion coefficient of $0.9 \times 10^{-6} / \mathrm{C}^{\circ}$. The tube is shielded with Al foils against the influence of humidity changes. A heat shield between this tube and the rocket skin isolates the rocket skin from the telescope. The temperature of the heat shield rose by $1^{\circ} \mathrm{C}$ during our last flight, while the temperature changes of the epoxy tube were smaller than $0.1^{\circ} \mathrm{C}$. We conclude that the thermal stability of our telescope is well within the requirements, even for a resolution below 0.1 arcsec.

Focussing of the telescope is a major effort. The depth of focus is considerably larger for visible light than for soft $x$-rays. We used the arrangement in Fig. 8. The coordinates of the test pattern in the film plane and its return image were measured using an indicator on the microscope. After a measurement series the thickness of a shim plate in the camera mount was adjusted to move the return image into focus at the film plane. With about 100 focus readings we were abie to focus the instrument to within $\pm 5 \mu \mathrm{m}$; this error is sufficiently small for a resolution of 0.1 arcsec.

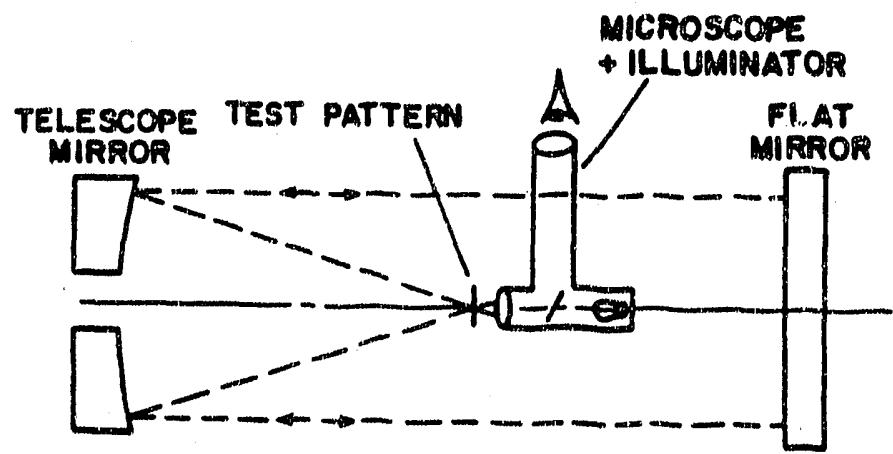

Fig. 8. Focussing of the telescope. A test pattern in the film plane is incoherently il. luminated and the microscope is focussed on the test pattern and the image of the test pattern returned from the lat mirror. 


\section{ROCKET POINTING STABILITY}

The "Solar Pointing Attitude Rocket Control System" (SPARCS) has been developed for solar observations from sounding rockets over the last three decades $[19,20]$. The fine sun sensor contains: two masks, one behind the other: the front mask has a square opening in the center and the back mask. has with 4 openings in the penumbra of the front mask, and a detector behind each opening. Perfect pointing is defined by equal intensity for each detector. A magnetometer and a Rate Integrating Gyro $(R I G)$ measure the roll of the spacecraft and jets of Freon gas are used for control. The specification for the system was \pm 1 arcsec/min until 1989 and it appears from our photos that the actual performance was better. Recent tests have shown a jitter well within 0.5 a icsec peak to peak and efforts to reach 0.1 arcsec are under way. It appears probable that the pointing stability will match the progress in the performance of telescopes for the next few years.

Free-flying spacecraft, such as the Hubble Space Telescope, use gyro assemblies for stabilization with the capabilicy of better than 0.01 arcsec fine-pointing stability [21]. Photon fux limitations will require the use of longer observation times and larger telescopes than are possible with sounding rockets for resolutions in the 0.01 arcsec range. We conclude therefore that the performance of future soft $x$-ray telescopes will not severely be limited by the available pointing stability.

\section{SUMMARY}

Normal incidence soft $\mathrm{x}$-ray telescopes with multilayer coatings have achieved sub-arcsecond resolution in photos of the corona of the sun from sounding rockets flights. Improvements in future flights can be expected. Resolutions better than 0.1 arcsec will require the use of satellites, where larger telescopes and longer observation times can provide the required number of photons. For the characterization of mirrors for diffraction limited performance it is necessary to measure deviations from the ideal surface with precision in the $1 \AA$-range for spatial periods covering the range from $10 \mathrm{~cm}$ to $1 \AA$, and we are presenting measurements over this range. The highest spatial frequencies affect mainily the reflectivity of the mirrors and several methods give results that are consistent with each other. It is much more difficult to measure the long spatial period $(\Lambda=1 \mathrm{~cm}$ to $10 \mathrm{~cm})$ figure errors with the same precision, and all our measurements in this range represent mainly the noise of the instruments and are upper limits on the figure errors of the optics. The performance of interferometers will have to be improved to reach the required precision.

Flights on sounding rockets will be limited to a resolution of 0.1 arcsec and it appears to be possible to maintain the mechanical tolerances on a telescope assembly throughout the launch and Right. Resolutions in the 0.01 arcsec range will require satellites with in-flight alignment (focussing) of the instrument. A comparison of the contrast of our photos with the measurements of the surface topography of the mirror surface demonstrates the reduction in the relative scattering from a inultilayer mirror compared to that of a single surface. 


\section{References}

[1] A. B. C. Walker, T. W. Barbee, R. B. Hoover, and J. F. Lindblom, "Soft X-ray images of the solar corona with a normal-incidenc: Cassegrain multilayer telescope," Science, vol. 241, pp. $1781.1787,1988$.

[2] L. Golcb, M. Herant, K. Kalata, I. Lovas, G. Nystrom, E. Spiller, and J. Wilczynski, " SubArcsecond Observations of the Solar X-ray Corona," Nature, vol. 344, pp. 842.844, 1990.

[3] E. L. Church, H. A. Jenkinson, and J. M. Zavada, "Relationship between surface scattering and microphotographic scattering," Opt. Engin., vol. 18:, pp. 125-136, 1979.

[4] E. L. Church and P. Z. Takacs, "The prediction of BRDFs from surface profile measurements," Proc. SPIE, vol. 1165:, pp. 136-150, 1989. BRDF = Bidirectional Reflectance Distribution Function

[5] Stearns D. G., "The Scattering of X-Rays from Nonideal Multilayer Structures," Journal of Applied Physics, vol. 65, pp. 491-506, 1989.

[6] J. E. Harvey, W. P. Zmek, and C. Ftaclas, "Imaging capabilities of normal incidence x-ray telescopes," Opt. Engin., vol. 29, pp. 603-608, 1990.

[7] P. Croce, L. Nevot, and B. Pardo, "Conuribution a l'étude des couches mince par réflexion spéculaire de rayon X," Nouv. Rev. d"Oprique appliquée, vol. 3, pp. 37.50, 1972.

[8] R. P. Haelbich, A. Segmuller, and E. Spiller, "Smooth Multilayer Films Suitabie for X-ray Mirrors," Appl. Phys. Lett., vol. 34:, pp. 184-186, 1979.

[9] E. Spiller and A. E. Rosenbiluth, "Determination of thickness errors and boundary roughness from the measured performance of a multilayer coating," Optical Engineering, vol. 25:, pp. 954-963, 1986.

[10] D. G. Stearns, M. B. Stearns, Y.Cheng, J. H. Stith, and N. M. Ceglio, "Thermally induced strucrural modifications of Mo.Si multilayers," J. Appl. Phys., vol. 67, pp. 2415-2427, 1990.

[11] K. Holloway, K. B. Do, and R. Sinclair, "Interfacial reactions on annealing molybdenum-silicon multilayers," J. Appl. Phy :, vol. 65, pp. 474-480, 1989.

[12] Nakajima K., Kita E., Koyano T., Fujiwara S., Tasaki A., and Aoki S., "Surface-Roughness Evaluation of Multilayer Coated X-Ray Mirrors by Scanning Tunneling Microscope," Japanese Journal of Applied Physics PART 2-LETTERS, vol. 28, pp. L854-L857, 1989.

[13] E. Spiller, "Enhancement of the reflectivity of multilayer $\mathrm{x}$-ray mirrors by Ion Polishing," $O p t$. Engin., vol. 29, pp. 609.613, 1990.

[14] S. R. Lange, "Very high resolution profiler fo diamond tuming groove analysis," Proc. SPIE, vol. 966, pp. $157-171,1988$.

[15] P. Z. Takacs, S. K. Feng, E. L. Church, S. N. Qiun, and W. M. Liu, "Long trace profile measurements on cylindical aspheres," Proc. SPIE, vol. 966:, pp. 354-364, 1988.

[16] B. E. 'Truax, "Absclute interferometric testing of spherical surfaces," Proc. SPIE, vol. 966, pp. 130-137, 1988.

[17] E. Spiller, K. Grebe, and L. Golub, "Filters for Soft X-Ray Solar Telescopes," Opt. Engin., vol. 29. pp. 625.631, 1990.

[18] L. Golub, G. Nystrom, E. Spiller, and J. Wilsczynski, "Construction of a Multilayered X-Ray Telescope for Solar Coronal Studies from Space," Proc. SPIE, vol. 563:, pp. 266-274, 1985.

[19] Q. M. Hansen, E. A. Gabris, M. D. Pearson, and B. S. Leonard, "A gyroless solar pointing attidude control systern for the Aerobee sounding rocket," J. Spacecraft, vol. 4, pp. 1443-1447, 1967.

[20] T. T. Tarshis and G. T. Sakoda, "A second generation sun sensor for sounding rocket applications," Soumding Rocket Technology Conference S, pp. 85.90, American Inst. Aeronatics and Astronautics inc., AlAA 79-0498, 1979.

[21] G. A. Beals, R. C. Crum, H. J. Dougherty, D. K. Hegel, J. L. Kelley, and J. J. Rodden, "Hubble space telescope precision pointing control systern," J. Guidance, vol. 11, pp. :19-123, 1988. 

